08

\title{
Электрофизические характеристики многослойных мемристивных наноструктур на основе стабилизированного иттрием диоксида циркония и оксида тантала
}

\author{
() С.В. Тихов, А.И. Белов, Д.С. Королев, И.Н. Антонов, А.А. Сушков, Д.А. Павлов, Д.И. Тетельбаум, \\ О.Н. Горшков, А.Н. Михайлов
}

Нижегородский государственный университет им. Н.И. Лобачевского, 603950 Нижний Новгород, Россия

e-mail: tikhov@phys.unn.ru

Поступило в Редакцию 21 мая 2019 г.

В окончательной редакции 21 мая 2019г.

Принята к публикации 10 июня 2019 г.

Изучены электрофизические характеристики многослойной мемристивной структуры $\mathrm{Au} / \mathrm{Ta} / \mathrm{ZrO}_{2}(\mathrm{Y}) / \mathrm{TaO}_{x} / \mathrm{TiN}$. Обнаружены электронные и ионные электретные эффекты, связанные с захватом носителей заряда на ловушки и ионной миграционной поляризацией в диэлектрике. Установлено влияние ловушек на процессы электроформовки и резистивного переключения. Определены значения энергии активации и концентрации для ловушек и ионов. Обнаружено явление стабилизации резистивного переключения, которое связывается с особенностями двуслойной структуры $\mathrm{TaO}_{x}$ и самоформирующимися нанокластерами Та. Нанокластеры выполняют роль концентраторов электрического поля в процессе электроформовки и последующего резистивного переключения.

Ключевые слова: мемристор, резистивное переключение, ловушки, ионы, миграционная поляризация, нанокластеры.

DOI: 10.21883/JTF.2020.02.48824.211-19

\section{Введение}

Важной научно-технической проблемой, которая сдерживает применение мемристивных устройств в электронных схемах на основе традиционных цифровых элементов (микросхемах памяти Resistive Random Access Memory - RRAM, гибридных аналого-цифровых прототипах нейронных сетей), является то, что процесс резистивного переключения имеет выраженную стохастическую природу. Это означает, что на каждом цикле переключения новое резистивное состояние соответствует новой конфигурации атомов в локальной области переключения. Связанный с этим разброс токов и напряжений резистивного переключения создает существенные трудности для реализации гибридных электронных схем на основе мемристоров. Поэтому значительные усилия направляются на проведение исследований и разработок, призванных повысить воспроизводимость параметров резистивного переключения [1].

Управление воспроизводимостью мемристивного эффекта может быть осуществлено путем формирования специальных концентраторов электрического поля [2], определяющих параметры проводящих каналов (филаментов), с разрушением и восстановлением которых связано резистивное переключение, а также путем выбора (инженерии) материалов в мемристивной структуре $[3,4]$. Используется также адаптивное программирование резистивного состояния путем коррекции параметров переключающих импульсов напряжения в зависимости от результата программирования [5-7]. Эти подходы не потеряли своей актуальности при проведении исследований и создании прототипов функциональных устройств на основе мемристоров, однако требуют усложнения технологического процесса создания мемристивных устройств и управляющих схем.

В настоящей работе с целью получения воспроизводимого резистивного переключения впервые изготовлена и исследована мемристивная структура, содержащая двуслойный диэлектрик $\mathrm{ZrO}_{2}(\mathrm{Y}) / \mathrm{TaO}_{x}$ и металлические нанокластеры, которые формируются в процессе роста структуры и выполняют роль концентраторов электрического поля при электроформовке и резистивном переключении. На основе предложенной структуры реализованы в интегральном исполнении массивы мемристивных микроустройств, которые демонстрируют стабильное резистивное переключение.

\section{Методика эксперимента}

Мемристивные структуры $\mathrm{Au} / \mathrm{Ta} / \mathrm{ZrO}_{2}(\mathrm{Y}) / \mathrm{TaO}_{x} / \mathrm{TiN}$ формировались на основе подложек окисленного кремния (толщина окисла $500 \mathrm{~nm}$ ) с проводящими слоями $\mathrm{Ti}(25 \mathrm{~nm})$ и $\mathrm{TiN}(25 \mathrm{~nm})$, полученными магнетронным методом в промышленных условиях. Слои $\mathrm{ZrO}_{2}(\mathrm{Y})$ и $\mathrm{TaO}_{x}$ с номинальной толщиной $10 \mathrm{~nm}$ были получены ВЧ-магнетронным распылением мишеней $\mathrm{ZrO}_{2}+12 \mathrm{~mol} . \% \mathrm{Y}_{2} \mathrm{O}_{3}$ и Та в смеси газов аргона и кислорода $(1: 1)$ на установке магнетронного распыления Torr 
International 2g1-1g2-eb4-th1 при температуре подложки $300^{\circ} \mathrm{C}$. На этой же установке при температуре $200^{\circ} \mathrm{C}$ магнетронным распылением в аргоне на диэлектрик наносились слои Та $(8 \mathrm{~nm})$, а затем $\mathrm{Au}(40 \mathrm{~nm})$ через маску с площадью отверстий $S \approx 10^{-3}$ и $10^{-2} \mathrm{~cm}^{2}$. Аналогичные мемристивные структуры были также реализованы в интегральных устройствах „кросс-пойнт“ с площадью $20 \times 20 \mu \mathrm{m}^{2}\left(S \approx 4 \cdot 10^{-6} \mathrm{~cm}^{2}\right)$ по оригинальной топологии [8], смонтированных в металлокерамическом корпусе.

На полученных структурах измерялись вольт-амперные (ВАХ), вольт-фарадные (ВФХ) и вольт-симменсные $(\mathrm{BCX})$ характеристики в интервале напряжений $0-10 \mathrm{~V}$ со скоростью пилообразной развертки по напряжению $\beta \approx 0.25-0.003 \mathrm{~V} / \mathrm{s}$. ВФХ и $\mathrm{BCX}$ измерялись при малом тестирующем напряжении $10 \mathrm{mV}$ с частотой $10^{3}-10^{6} \mathrm{~Hz}$. Знак управляющего напряжения определялся потенциалом на верхнем электроде мемристивной структуры $(\mathrm{Au} / \mathrm{Ta})$. Измерялись также малосигнальные характеристики мемристивных структур (тестовое напряжение $10 \mathrm{mV}$ ) в параллельной и последовательной схемах замещения $[9,10]$ в интервале частот $f=10^{3}-10^{7} \mathrm{~Hz}$. Измерения характеристик проводились в исходном состоянии (IS), состояниях после электроформовки (FOR), после переключения в состояние с высоким сопротивлением (HRS) и состояние с низким сопротивлением (LRS). Определялись величины параллельной емкости $\left(C_{p}\right)$; параллельной проводимости, приведенной к круговой частоте $\left(G_{p} / \omega\right)$; параллельного сопротивления $\left(R_{p}\right)$; последовательного сопротивления $\left(R_{s}\right)$; тангенса угла диэлектрических потерь $(\operatorname{tg} \delta)$. Изучалось влияние количества переключений при подаче импульсов отрицательной и положительной полярности с длительностью $10^{-3} \mathrm{~s}$ на значение тока через структуру при тестирующем напряжении $0.3 \mathrm{~V}$.

Для температурных измерений образцы помещались в герметически закрытый металлический термостат. Измерения осуществлялись на воздухе в интервале температур $77-510 \mathrm{~K}$ с использованием изотермического режима измерений. Температура поддерживалась с точностью до $1 \mathrm{~K}$. Все электрофизические измерения осуществлялись на анализаторе полупроводников приборов Agilent B1500A.

\section{Результаты и обсуждение}

Методом просвечивающей электронной микроскопии высокого разрешения было установлено, что пленка $\mathrm{TaO}_{x}$ является аморфной, а пленка $\mathrm{ZrO}_{2}(\mathrm{Y})$ характеризуется колончатой поликристаллической структурой (рис. 1). Анализ состава структуры показал, что на границе $\mathrm{TaO}_{x} / \mathrm{TiN}$ при осаждении происходит частичное замещение атомов азота на атомы кислорода с формированием интерфейсного поликристаллического слоя $\mathrm{TiO}_{2}$ и слоя нанокластеров Та с размерами $2-3 \mathrm{~nm}$ (стрелки на рис. 1). Переходный слой из окисленного Та

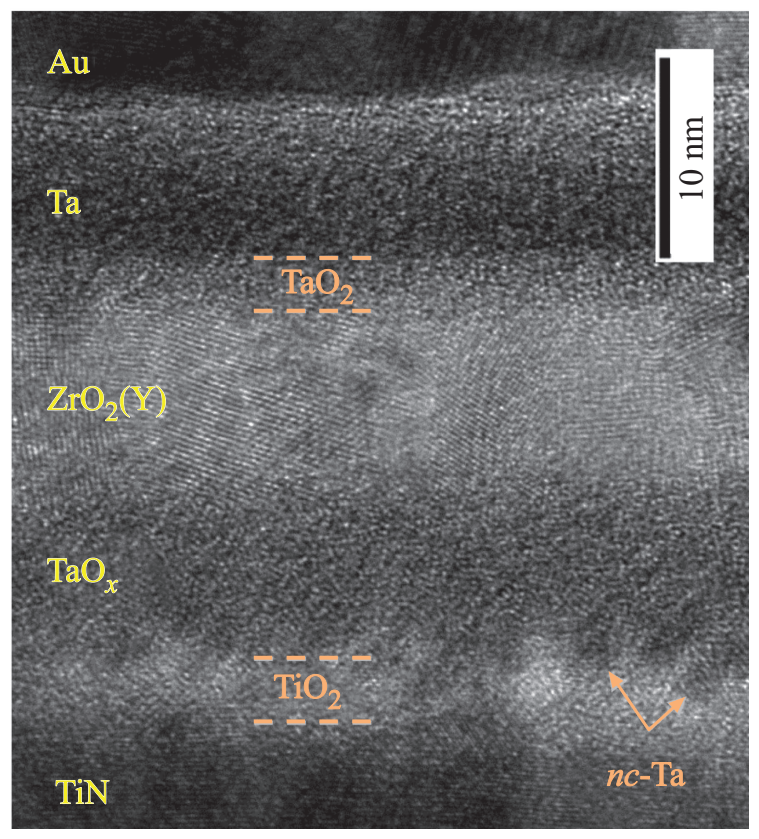

Рис. 1. Изображение просвечивающей электронной микроскопии поперечного сечения мемристивной наноструктуры $\mathrm{Au} / \mathrm{Ta} / \mathrm{ZrO}_{2}(\mathrm{Y}) / \mathrm{TaO}_{x} / \mathrm{TiN}$.

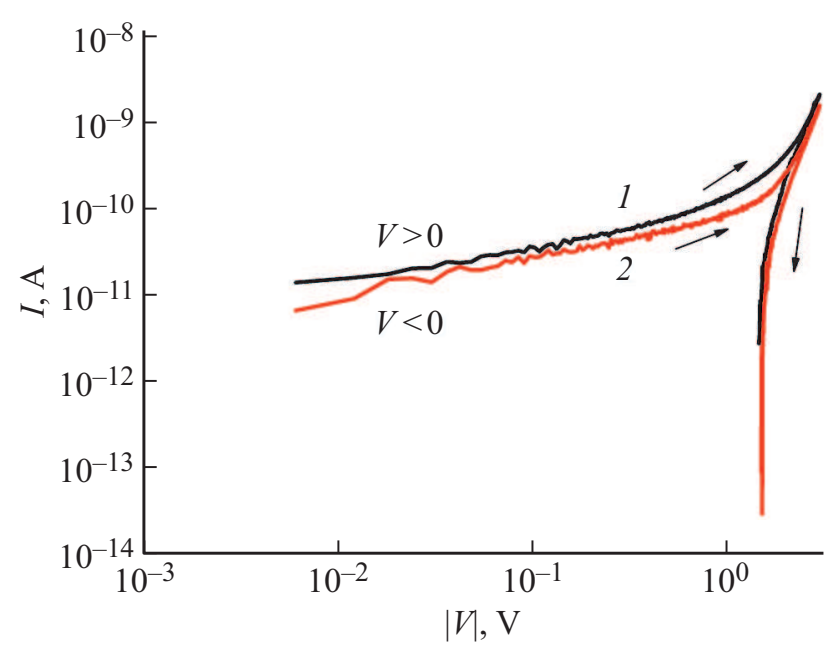

Pис. 2. BAX мемристивной структуры в IS: $1-$ при подаче на верхний электрод положительного смещения $V>0,2-$ при подаче отрицательного смещения $V<0 ; \beta \approx 0.12 \mathrm{~V} / \mathrm{s}$, $S \approx 1 \cdot 10^{-3} \mathrm{~cm}^{-2}$.

формируется на интерфейсе между $\mathrm{ZrO}_{2}(\mathrm{Y})$ и верхним электродом, создавая избыток кислородных вакансий в $\mathrm{ZrO}_{2}(\mathrm{Y})$.

B IS структуры наблюдался гистерезис BAX (рис. 2) и электретный эффект (ЭЭ) [9]. Ток I уменьшался при обратном ходе напряжения $V$ вплоть до смены знака и оставался при снятом внешнем напряжении. Этот гистерезис проявлялся уже при комнатной температуре и может быть объяснен захватом свободных электронов на глубокие ловушки, локализованные в диэлектрике на 


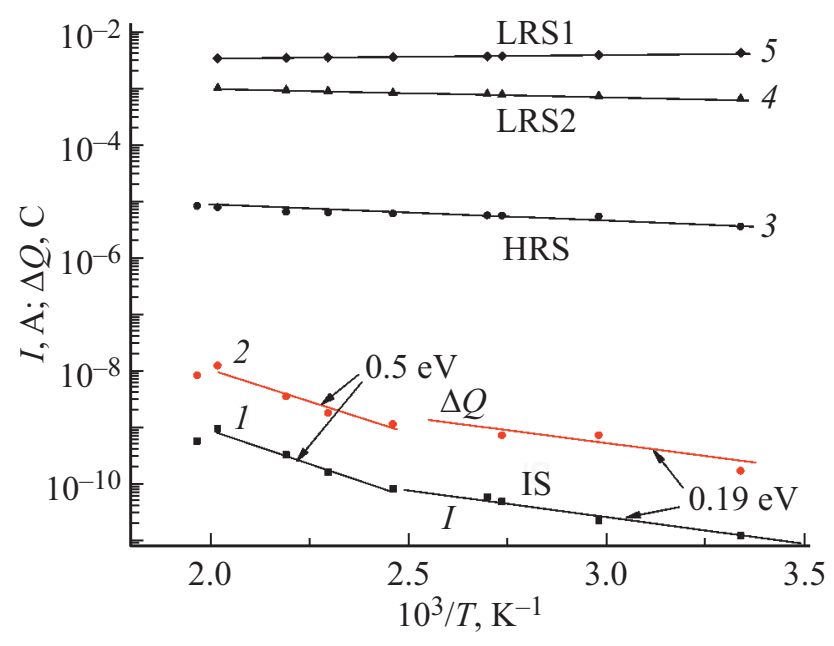

Рис. 3. Зависимости $I(1,3-5)$ и $\Delta Q$ (2) от обратной температуры в состояниях IS $(1,2)$, HRS (3), в состоянии LRS2 с нелинейной BAX (4) и в состоянии LRS1 с линейной $\operatorname{BAX}(5) ; S \approx 1 \cdot 10^{-3} \mathrm{~cm}^{-2}$.

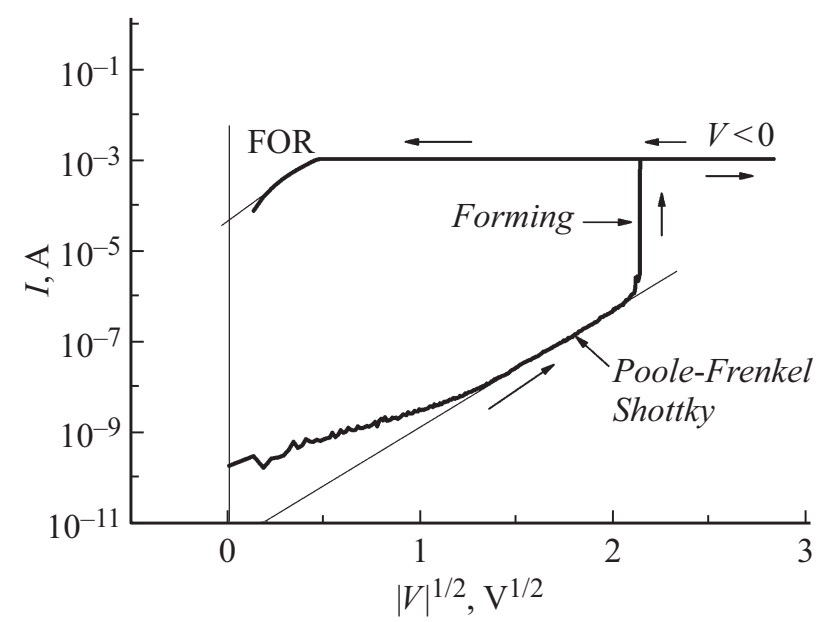

Рис. 4. ВАХ, иллюстрирующая формовку при ограничении тока величиной $1 \mathrm{~mA}$, и достижение состояния FOR в мемристивной структуре с площадью $S \approx 1 \cdot 10^{-2} \mathrm{~cm}^{-2}$.

границе с металлической обкладкой, переходом части этого заряда на металл и возникновением электрического поля в диэлектрике, создающего в нем ток при отсутствии внешнего напряжения. Поверхностная концентрация этих ловушек $N_{t}$, согласно [11], может быть оценена по площади петли гистерезиса $\mathrm{BAX} \Delta Q$ из соотношений

$$
\Delta Q=\int_{0}^{V} \frac{I d V}{\beta}-\int_{V}^{0} \frac{I d V}{\beta}
$$

и

$$
N_{t}=\frac{\Delta Q}{q \cdot S}
$$

где $q$ - заряд электрона, $\beta$ - скорость развертки по напряжению.
Из анализа температурных зависимостей $I$ и $\Delta Q$ в IS, приведенных на рис. 3 (кривые 1,2), следует, что с увеличением температуры выше $410 \mathrm{~K}$ (измерения проводились в интервале $300-510 \mathrm{~K}$ ) электронный ЭЭ переходил в ионный ЭЭ. На это указывает и то, что в указанном температурном интервале с ростом температуры был обнаружен рост квазистатической емкости из-за миграционной поляризации ионов [11]. Энергия активации первого эффекта составляет около $0.19 \mathrm{eV}$, а второго $-0.5 \mathrm{eV}$. Значение $0.5 \mathrm{eV}$ хорошо соответствует энергии активации миграции вакансий кислорода по границам зерен в $\mathrm{ZrO}_{2}(\mathrm{Y})$ [11]. Из температурной зависимости петли ионного гистерезиса (рис. 3, кривая 2) по формулам (1), (2) были оценены максимальные измеренные поверхностные концентрации ловушек $\left(\sim 10^{13} \mathrm{~cm}^{-2}\right)$ при температуре $400 \mathrm{~K}$ и ионов

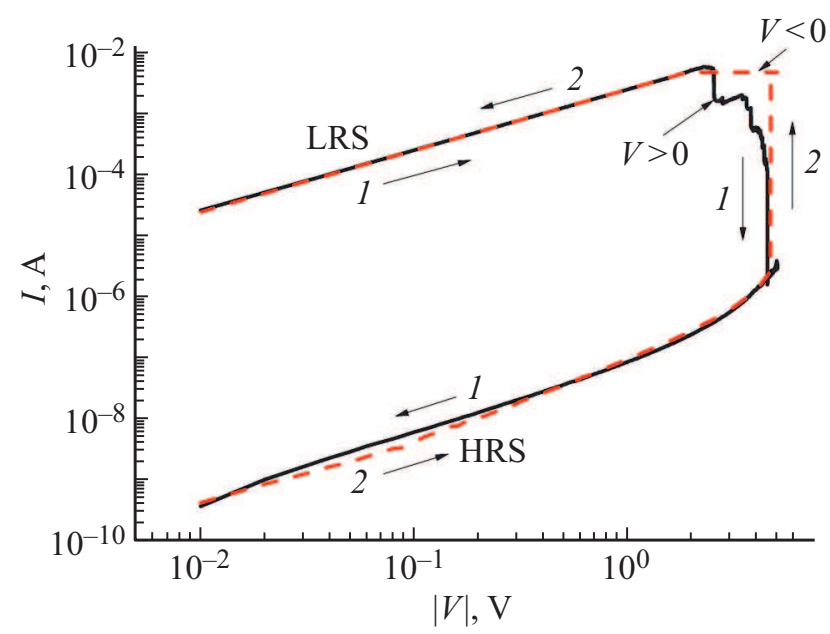

Рис. 5. ВАХ, демонстрирующая первое переключение после ЭФ. Сплошная кривая соответствует развертке напряжения при $V>0$ на электроде $\mathrm{Au,} \mathrm{штриховая} \mathrm{кривая} \mathrm{-} \mathrm{развертке}$ при $V<0$. Последовательность подачи напряжения указана стрелками. $\beta \approx 0.34 \mathrm{~V} / \mathrm{s}$.

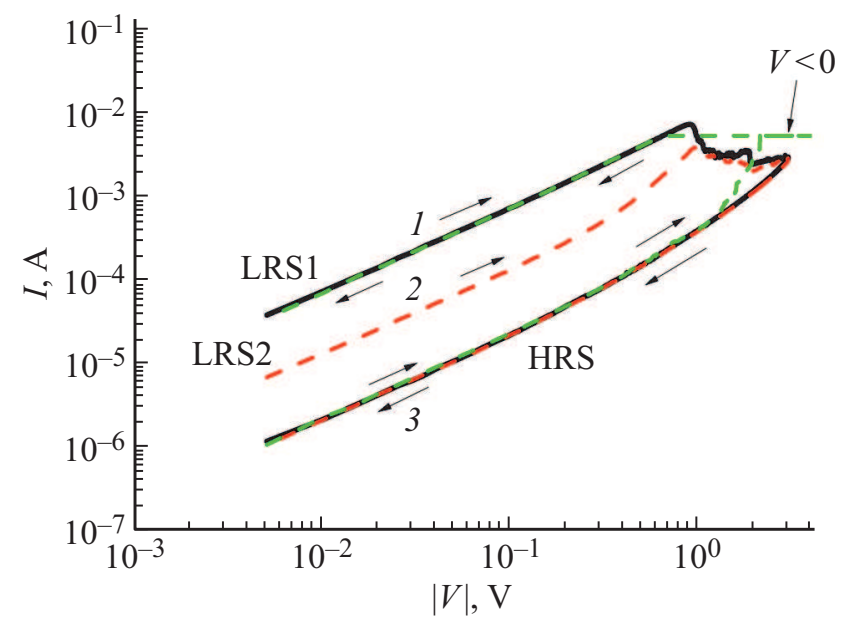

Рис. 6. Типичные ВАХ мемристивной структуры в ходе первых 100 циклов переключения. $\beta \approx 0.34 \mathrm{~V} / \mathrm{s}$. 


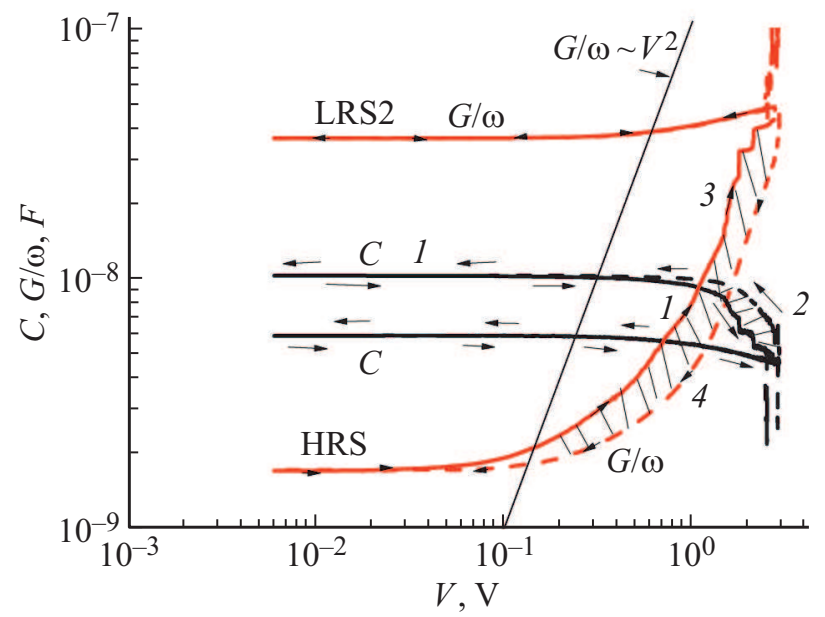

Рис. 7. Зависимости емкости $C(1,2)$ и активной проводимости, приведенной к круговой частоте $G / \omega(3,4)$, от $V$ на частоте $50 \mathrm{kHz}\left(S \approx 1 \cdot 10^{-2} \mathrm{~cm}^{-2}, \beta \approx 0.2 \mathrm{~V} / \mathrm{s}\right)$. Сплошные линии получены при $V<0$, штриховые - при $V>0$.

$\left(\sim 10^{15} \mathrm{~cm}^{-2}\right)$ при температуре $\sim 510 \mathrm{~K}$, участвующих в поляризации (в случае ионного ЭЭ поверхностная плотность заряда в выражении (2) определяется концентрацией ионов).

При осуществлении электроформовки (ЭФ) с ростом отрицательного напряжения на BAX наблюдался сначала сублинейный рост тока (рис. 4), связанный с заполнением ловушек. Затем следовал линейный участок $I \sim V$, а за ним - участок более резкого роста тока (рис. 4), на котором $\log I \sim V^{1 / 2}$. Последняя зависимость характерна для эффектов Шоттки или Пула-Френкеля (эти эффекты в нашем случае трудно различить из-за двуслойности диэлектрика). Оценка по ВАХ, построенной в координатах Шоттки [12], дала значение высоты барьера для электронов на границе Ta/диэлектрик $\sim 1 \mathrm{eV}$. В конце экспоненциального участка ВАX локальная температура в области концентрации электрического поля из-за нагрева током могла достигать значения, необходимого для образования подвижных ионов кислорода, после чего скачком (участок вертикального роста на рис. 4) происходила ЭФ путем выстраивания филаментов из ионных цепочек. Ток при ЭФ не зависел от площади структуры, что указывает на возникновение филаментов с одинаковой токонесущей способностью на значительно меньших площадях.

После ЭФ ЭЭ исчезал, и структура демонстрировала биполярное резистивное переключение в диапазоне напряжений $\pm 5 \mathrm{~V}$ (рис. 5), характерное для структур на основе оксидов металлов с эффектом изменения валентности (valence change mechanism) [13]. На начальном этапе измерений (в ходе первых переключений) имели место большое отношение токов в состояниях LRS и HRS (более четырех порядков величины) и значительный разброс значений этих параметров. По мере увеличения числа циклов переключения происходили изменения в параметрах резистивного переключения (типичные BAX после первых 100 циклов переключения приведены на рис. 6). В частности, наблюдалось уменьшение отношений токов в состояниях LRS и HRS. Кроме того, происходила смена характера проводимости в низкоомном состоянии. Если в HRS всегда наблюдается нелинейная BAX (кривая 3), то в LRS для разных циклов наблюдается как линейная, так и нелинейная зависимости тока от напряжения (кривые 1 и 2, помеченные как LRS1 и LRS2 соответственно).

Температурные зависимости тока для указанных состояний приведены на рис. 3 в сравнении с аналогичной зависимостью для IS (до ЭФ). Нелинейные состояния HRS и LRS2 характеризовались относительно слабой температурной зависимостью, имеющей полупроводниковый характер. Температурная зависимость тока в LRS1 (с линейной зависимостью $\mathrm{BAX)}$ характеризовалась положительным температурным коэффициентом сопротивления $\alpha$ с характерным для большинства металлов значением $\sim 10^{-3} \mathrm{~K}\left(\alpha \approx 1.17 \cdot 10^{-3} \mathrm{~K}^{-1}\right)$. Однако это значение меньше, чем для тантала $\left(3.8 \cdot 10^{-3} \mathrm{~K}^{-1}\right)$ и золота $\left(4 \cdot 10^{-3} \mathrm{~K}^{-1}\right)$ [14], что можно связать с тем, что проводимость реализуется по филаменту, который образован кислородными вакансиями в межзеренной границе $\mathrm{ZrO}_{2}(\mathrm{Y})[13,14]$. Для состояния LRS2 характерен полупроводниковый характер протекания тока, а степенная зависимость от температуры характеризуется показателем степени, зависящим от величины напряжения и увеличивающимся в пределах от 0.5 до 2 с уменьшением напряжения. Такое поведение может наблюдаться при частичном шунтировании нелинейного канала протекания тока параллельным омическим сопротивлением.

После нескольких сотен циклов переключения ВАХ стабилизировалась: переключение происходило между HRS и промежуточным состоянием LRS2 (кривые 2 и 3 на рис. 6) и характеризовалось низким разбросом значений токов от цикла к циклу. Наблюдаемый эф-

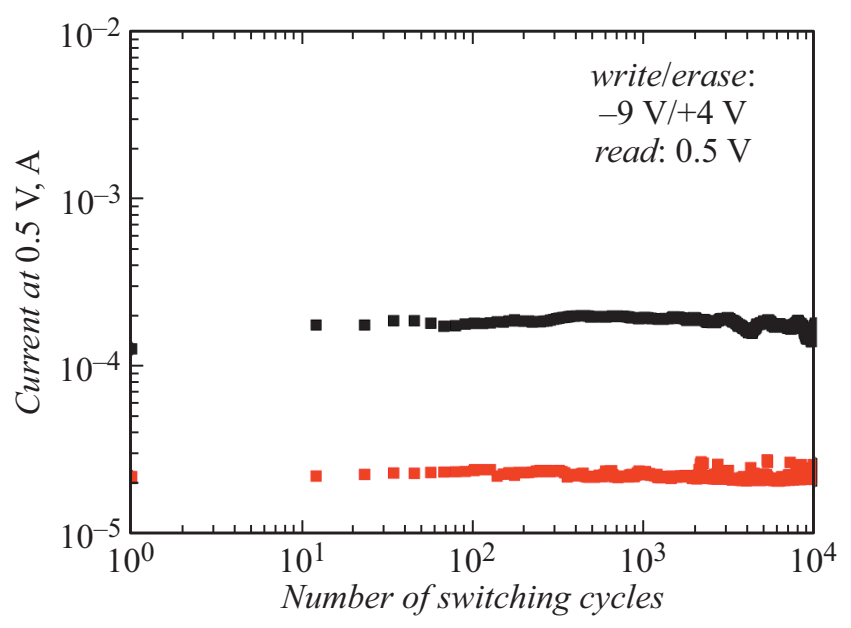

Рис. 8. Данные по стабильности резистивного переключения в импульсном режиме для мемристивной структуры в интегральном (корпусном) исполнении. 
Параметры эквивалентной цепи мемристивных структур в различных состояниях

\begin{tabular}{c|l|c|c|c|c|c|c}
\hline № & Состояние & $C_{0}, \mathrm{~F}$ & $\operatorname{tg} \delta_{0}$ & $\operatorname{tg} \delta_{\infty}$ & $R_{p 0}, \Omega$ & $R_{s \infty}, \Omega$ & $\tau=R_{s \infty} \cdot C_{0}, \mathrm{~s}$ \\
\hline \multicolumn{7}{c}{ Структура с площадью $\sim 10^{-2} \mathrm{~cm}^{2}$} \\
\hline 1 & IS & $1.12 \cdot 10^{-8}$ & 0.017 & 1.89 & $8.27 \cdot 10^{5}$ & 20.5 & $2.3 \cdot 10^{-7}$ \\
2 & FOR & $6.16 \cdot 10^{-9}$ & 285 & 2.45 & 90.5 & 21.0 & $1.3 \cdot 10^{-7}$ \\
3 & HRS & $7.85 \cdot 10^{-9}$ & 45 & 2.00 & 490 & 44.0 & $3.4 \cdot 10^{-7}$ \\
4 & LRS2 & $5.27 \cdot 10^{-9}$ & 215 & 2.00 & 157 & 44.0 & $2.3 \cdot 10^{-7}$ \\
5 & LRS1 & $8.17 \cdot 10^{-8}$ & 1450 & 4.40 & 62 & 44.0 & $3.6 \cdot 10^{-7}$ \\
\hline
\end{tabular}

Структура с площадью $4 \cdot 10^{-6} \mathrm{~cm}^{2}$

\begin{tabular}{l|l|l|l|l|l|l|l|}
\hline 6 & HRS & $1.00 \cdot 10^{-11}$ & 100 & 0.8 & $1.00 \cdot 10^{5}$ & - \\
7 & LRS & $2.00 \cdot 10^{-11}$ & 1000 & 1.0 & $9.00 \cdot 10^{3}$ & -
\end{tabular}

фект может быть связан с образованием филаментов со стабильными геометрическими параметрами и нелинейной проводимостью в области самоформирующихся нанокластеров Та - концентраторов электрического поля (рис. 1). В работе [15] также делается вывод, что наличие металлических частиц в областях формирования проводящих филаментов значительно увеличивает устойчивость системы к многократному переключению.

Следует отметить, что контрольные мемристивные структуры $\mathrm{Au} / \mathrm{Ta} / \mathrm{ZrO}_{2}(\mathrm{Y}) / \mathrm{TiN}$, не содержавшие слоя оксида тантала, не демонстрировали эффект стабилизации переключения. По-видимому, слой $\mathrm{TaO}_{x}$ также играет важную роль в процессе трансформации геометрических параметров и проводимости филаментов в двуслойном диэлектрике $\mathrm{ZrO}_{2}(\mathrm{Y}) / \mathrm{TaO}_{x}$, так как джоулев разогрев и изменение стехиометрии могут вызывать воспроизводимые структурно-фазовые превращения в слое оксиде тантала между более проводящей рутилоподобной фазой $\mathrm{TaO}_{2}$ и диэлектрической фазой $\mathrm{Ta}_{2} \mathrm{O}_{5}[16]$. Вклад данных превращений в наблюдаемое резистивное переключение подлежит отдельному экспериментальному и теоретическому исследованию.

Для выяснения причин нелинейностей BAX в состояниях HRS и LRS2 были измерены зависимости малосигнальных емкости $C$ и приведенной к круговой частоте проводимости $G / \omega$ от $V$, которые также обнаружили нелинейное поведение. Эти зависимости приведены на рис. 7. Максимумы на зависимостях $G / \omega$ от $V$ и сдвиг ВФХ и ВСХ по оси $V$ (ср. кривые 1,2 и 3, 4 на рис. 7) связаны с влиянием захвата электронов из верхнего слоя Та при $V<0$ на ловушки в диэлектрике [17]. При наличии порогового напряжения данный процесс происходит параллельно с трансформацией филаментов. При этом в диэлектрике образуется встроенное электрическое поле со знаком „минус“ у Та и „плюс“ у TiN. В связи с этим величина порогового напряжения $V>0$ для переключения структуры в HRS увеличивается. Оценка концентрация ловушек по площади под кривыми сдвига (заштрихованы на рис. 7) и по максимумам на кривых проводимости с использованием выражения [17]:

$$
G_{\max } / \omega=0.4 q^{2} N_{t} S
$$

дала суммарное значение $\sim 10^{13} \mathrm{~cm}^{-2}$.

Наблюдающаяся при комнатной температуре нелинейная и близкая к квадратичной зависимость $G / \omega$ от $V$ (рис. 7, кривые 3 и 4), вероятно, связана с изменением токонесущей способности (геометрии) филаментов в процессе их трансформации [18]. При температуре жидкого азота $77 \mathrm{~K}$ нелинейность несколько сглаживалась (зависимость оказывалась слабее квадратичной), что также может быть связано с уменьшением токонесущей способности филаментов при этой температуре. При температуре $77 \mathrm{~K}$ существенно уменьшалось влияние ловушек на кривые переключения емкости и проводимости из-за уменьшения вероятности их перезарядки.

Мемристивная структура, изготовленная по технологии „,кросс-пойнт“ с площадью $4 \cdot 10^{-6} \mathrm{~cm}^{2}$ в интегральном исполнении, также проявляла стабильные резистивные переключения - более $10^{4}$ циклов переключений (рис. 8). Отличие в значениях тока и других параметров переключения может быть связано с иными значениями площади структуры, дополнительными сопротивлениями токоподводящих элементов и особенностями технологических процессов при изготовлении микроустройств.

В таблице приведены сводные данные по параметрам эквивалентной цепи для мемристивной структуры в IS, после формовки (FOR), в состояниях HRS, LRS1 и LRS2, а также для мемристивного микроустройства „кросс-пойнт“. Индекс 0 обозначает частоту тестирующего сигнала $10^{3} \mathrm{~Hz}$, индекс $\infty-10^{6} \mathrm{~Hz}$. В строках $1-5$ приведены параметры для разных состояний мемристивной структуры с площадью $\sim 10^{-2} \mathrm{~cm}^{2}$, а в строках 6,7 - для мемристивной структуры в интегральном исполнении с площадью $4 \cdot 10^{-6} \mathrm{~cm}^{2}$.

Во всех случаях в проводящем состоянии значение $\operatorname{tg} \delta_{0}$ много больше значения $\operatorname{tg} \delta_{\infty}$. Такое поведение характерно для омических потерь, связанных с образованием филаментов $[10,13,19]$. Различие отношения 
величин $\operatorname{tg} \delta$ в высокоомном и низкоомном состоянии достигало 5 порядков величины на частоте $10^{3} \mathrm{~Hz}$. Эти отношения значительно различались при каждом новом переключении. То же самое можно сказать о достигаемых значениях величины $R_{p 0}$, которая определяет шунтирующее сопротивление структуры [19-21]. После стабилизации переключения эти отношения значительно уменьшались и также стабилизировались (строки 3, 4 в таблице). Следует подчеркнуть независимость величины $R_{s \infty}$ от состояния мемристора после стабилизации переключения, которое при больших частотах определяется сопротивлением верхнего электрода и свидетельствует об отсутствии ярко выраженных электрохимических процессов на этом электроде. Время считывания параметров эквивалентной схемы мемристора $\tau=R_{s \infty} \cdot C_{0}[20,21]$ и сопротивление $R_{s \infty}$ удавалось определить только для структур с относительно большой площадью. Значения $\tau$, как видно из таблицы, не сильно отличались для разных состояний таких структур. Величину $R_{s} \infty$ для структур типа „кросс-пойнт“ не удавалось определить из-за отсутствия насыщения этой величины в исследованном диапазоне частот.

Следует отметить, что мемристивные структуры в интегральном (корпусном) исполнении не требуют операции формовки, сразу обратимо переключаются на порядок по $\operatorname{tg} \delta$ и сопротивлению $R_{p 0}$ (строки 6,7$)$ и демонстрируют стабильное переключение между нелинейными резистивными состояниями, важное для их применения в нейроморфных системах.

\section{Заключение}

В разработанной мемристивной структуре $\mathrm{Au} / \mathrm{Ta} / \mathrm{ZrO}_{2}(\mathrm{Y}) / \mathrm{TaO}_{x} / \mathrm{TiN}$ до электроформовки в высокоомном исходном состоянии обнаружены электретные эффекты, связанные с захватом электронов на ловушки в диэлектрике и токами ионной миграционной поляризации. Определены значения энергии активации для этих процессов, а также оценены концентрации участвующих в них ловушек и ионов. В структурах после электроформовки при переключении обнаружена нелинейность вольт-фарадных и вольт-симменсных характеристик, обусловленная влиянием ловушек и изменением токонесущей способности филамента. После нескольких сотен циклов переключения обнаружена стабилизация переключения между нелинейными состояниями, которые характеризуются низким разбросом значений сопротивления. Стабилизация параметров резистивного переключения может быть интерпретирована на основе представлений о формировании филаментов, локализованных в пленке $\mathrm{ZrO}_{2}(\mathrm{Y})$ по границам зерен, наличием нанокластеров Та как концентраторов электрического поля в пленке $\mathrm{TaO}_{x}$ и воспроизводимых структурных превращениях в пленке оксида тантала с формированием более проводящей и диэлектрической фаз.

\section{Финансирование работы}

Работа выполнена в рамках гранта Российского фонда фундаментальных исследований (РФФИ 18-29-23001).

\section{Конфоликт интересов}

Авторы заявляют, что у них нет конфликта интересов.

\section{Список литературы}

[1] Ielmini D. // Semicond. Sci. Technol. 2016. Vol. 31. P. 063002. DOI: 10.1088/0268-1242/31/6/063002

[2] Sun Y., Song C., Yin J., Chen X., Wan Q., Zeng F., Pan F. // ACS Appl. Mater. Interf. 2017. Vol. 9. P. 34064-34070. DOI: $10.1021 /$ acsami.7b09710

[3] Trapatseli M., Cortese S., Serb A., Khiat A., Prodromakis T. // J. Appl. Phys. 2017. Vol. 121. P. 184505-1-184505-8. DOI: $10.1063 / 1.4983006$

[4] $W u W$. ., Wu H., Gao B., Deng N., Qian H. // J. Appl. Phys. 2018. Vol. 124. P. 152108-1-152108-6. DOI: $10.1063 / 1.5037896$

[5] Alibart F., Gao L., Hoskins B.D., Strukov D.B. // Nanotechnology. 2012. Vol. 23. P. 075201-1-075201-7. DOI: 10.1088/0957-4484/23/7/075201

[6] Berdan R., Prodromakis T., Toumazou C. // Electron. Lett. 2012. Vol. 48. N 18. P. 1105-1107. DOI: $10.1049 / \mathrm{el} .2012 .2295$

[7] Никируй К.Э., Емельянов А.В., Демин В.А., Рыльков В.В., Ситников А.В., Кашкаров П.К. // Письма в ЖТФ. 2018. T. 44. Вып. 10. C. 20-28.[Nikiruy K.E., Emelyanov A.V., Demin V.A., Rylkov V.V., Sitnikov A.V., Kashkarov P.K. // Tech. Phys. Lett. 2018. Vol. 44. N 5. P. 416-419. DOI: $10.1134 / \mathrm{S} 106378501805022 \mathrm{X}]$

[8] Свидетельство о государственной регистрации топологии интегральной микросхемы № 2018630129. Топология тестового кристалла с матрицей мемристивных микроустройств / Грязнов Е.Г., Антонов И.Н., Котина А.Ю., Котомина В.Е., Михайлов А.Н., Шарапов А.Н., Гориков О.Н. № 2018630123; заявлено 12.07.2018; опубл. 08.08.2018. $1 \mathrm{c}$.

[9] Орешкин П.Т. Физика полупроводников и диэлектриков. М.: Высшая школа, 1977. 448 с.

[10] Тихов С.В., Горшков О.Н., Антонов И.Н., Касаткин А.П., Королев Д.С., Белов А.И., Михайлов А.Н., Тетельбаум Д.И. // ЖТФ. 2016. Т. 86. Вып. 5. С. 107-111. [Tikhov S.V., Gorshkov O.N., Antonov I.N., Kasatkin A.P., Korolev D.S., Belov A.I., Mikhaylov A.N., Tetelbaum D.I. // Tech. Phys. 2016. Vol. 61. N 5. P. 745-749. DOI: $10.1134 / \mathrm{S} 106378421605025 \mathrm{X}]$

[11] Tikhov S., Gorshkov O., Antonov I., Morozov A., Koryazhkina M., Filatov D. // Adv. Mater. Sci. Eng. 2018. Vol. 2018. P. 2028491-1-2028491-8. DOI: 10.1155/2018/2028491

[12] Chiu F.-C., Lin Z.-H., Chang C.-W., Wang C.-C., Chuang K.F., Huang C.-Y., Lee J. Yamin, Hwang H.L. // J. Appl. Phys. 2005. Vol. 97. P. 034506-1-034506-4. DOI: $10.1063 / 1.1846131$ 
[13] Mikhaylov A.N., Gryaznov E.G., Belov A.I., Korolev D.S., Sharapov A.N., Guseinov D.V., Tetelbaum D.I., Tikhov S.V., Malekhonova N.V., Bobrov A.I., Pavlov D.A., Gerasimova S.A., Kazantsev V.B., Agudov N.V., Dubkov A.A., Rosário C.M.M., Sobolev N.A., Spagnolo B. // Phys. Status Solidi C. 2016. Vol. 13. P. 870-881.

DOI: $10.1002 /$ pssc. 201600083

[14] Электротехнический справочник / Под ред. В.Г. Герасимова. М.: МАИ, 2004. Т. 4.

[15] Рыльков В.В., Николаев С.Н., Демин В.А., Емельянов А.В., Ситников А.В., Никируй К.Э., Леванов В.А., Пресняков М.Ю., Талденков А.Н., Васильев А.Л., Черноглазов К.Ю., Веденеев А.С., Калинин Ю.Е., Грановский А.Б., Тугушев В.В., Бугаев А.С. // ЖЭТФ. 2018. Т. 153. Вып. 3. С. 424-441. [Rylkov V.V., Nikolaev S.N., Demin V.A., Emelyanov A.V., Sitnikov A.V., Nikiruy K.E., Levanov V.A., Presnyakov M.Yu., Taldenkov A.N., Vasiliev A.L., Chernoglazov K.Yu., Vedeneev A.S., Kalinin Yu.E., Granovsky A.B., Tugushev V.V., Bugaev A.S. // JETP. 2018. Vol. 126. N 3. P. 353-367. DOI: $10.1134 / \mathrm{S} 1063776118020152]$

[16] Garg S.P., Krishnamurthy N., Awasthi A., Venkataram M. / J. Phase Equilibria: Phase Diagram Eval. Sec. II. 1996. Vol. 17. N 1. P. $63-77$. DOI: $10.1007 / \mathrm{BF} 02648373$

[17] Возмилова Л.Н., Гаман В.И., Калыгина В.М., Панин А.В., Смирнова Т.П. // ФТП. 1997. Т. 31. Вып. 4. С. 492-497. [Vozmilova L.N., Gaman V.I., Kalygina V.M., Panin A.V., Smirnova T.P. // Semiconductors. 1997. Vol. 31. N 4. P. 418-422. DOI: $10.1134 / 1.1187175]$

[18] Ielmini D. // IEEE Tr. Electron. Dev. 2011. Vol. 58. N 12. P. 4309-4317. DOI: 10.1109/TED.2011.2167513

[19] Mikhaylov A.N., Belov A.I., Guseinov D.V., Korolev D.S., Antonov I.N., Efimovykh D.V., Tikhov S.V., Kasatkin A.P., Gorshkov O.N., Tetelbaum D.I., Bobrov A.I., Malekhonova N.V., Pavlov D.A., Gryaznov E.G., Yatmanov A.P. // Mater. Sci. Eng. B. 2015. Vol. 195. P. 48-54.

DOI: $10.1016 /$ j.mseb.2014.12.029

[20] Tikhov S.V., Mikhaylov A.N., Belov A.I., Korolev D.S., Antonov I.N., Karzanov V.V., Gorshkov O.N., Tetelbaum D.I., Karakolis P., Dimitrakis P. // Microelectron. Eng. 2018. Vol. 188. N 187. P. 134-138. DOI: 10.1016/j.mee.2017.11.002

[21] Тихов С.В., Гориков О.Н., Антонов И.Н., Тетельбаум Д.И., Михайлов А.Н., Белов А.И., Морозов А.И., Karakolis P., Dimitrakis P. // ФТП. 2018. Т. 52. Вып. 12. C. 1436-1442. DOI: 10.21883/FTP.2018.12.46753.32

[Tikhov S.V., Gorshkov O.N., Antonov I.N., Tetelbaum D.I., Mikhaylov A.N., Belov A.I., Morozov A.I., Karakolis P., Dimitrakis P. // Semiconductors. 2018. Vol. 52. N 12. P. 1540-1546. DOI: 10.1134/S1063782618120242] 